\title{
Rapid neurological recovery following delayed correction of I4-15 traumatic spondylolistheis
}

\begin{abstract}
We present in our article a case report of a patient who presented to us with bilateral foot drop secondary to L4-5 traumatic spondylolisthesis that occurred 3 months prior to her presentation to our hospital. She was treated surgically in two stages, the first stage included a wide laminectomy with bilateral facetectomies, followed by a progressive reduction of the listhesis and posterior pedicle screw fixation. The second stage followed which include anterior column support using a cage at L4-5 level. The patient showed remarkable recovery of her neurological status that started at the second postoperative day and had almost complete recovery after around 6 weeks. In case of traumatic spondylolisthesis with neurological compromise, surgical decompression and stabilization should be considered, even in late presentation, because neurological recovery is possible.
\end{abstract}

Keywords:Traumatic spondylolisthesis, Delayed correction, Neurological recovery
Volume 7 Issue 2 - 2017

\author{
Mohammad Badra,' Ammar Chemiassani, \\ Karam Karam, ${ }^{3}$ Tarek Sunna ${ }^{2}$ \\ 'Department of Orthopedic Surgery, Clemenceau Medical \\ Center, Lebanon \\ ${ }^{2}$ Department of Neurological Surgery, Lebanese University, \\ Lebanon \\ ${ }^{3}$ Department of Orthopedic Surgery, Lebanese American \\ University, Lebanon
}

Correspondence: Mohammad Ibrahim Badra, Department of Orthopedic Surgery, Clemenceau Medical Center, PO Box I I2555 Beirut-Lebanon Email mbadral3@gmail.com

Received: September 27, 2016 | Published: February 02, 2017

\section{Introduction}

Traumatic lumbar spondylolisthesis is considered to be a rare entity that results from a high-energy trauma. The mechanism of injury is thought to be a hyper-flexion type along with compression. ${ }^{1}$ The most common location of traumatic lumbar spondylolisthesis is at L5-S1 level secondary to the coronal facet orientation at that level. Several cases of traumatic dislocation at higher levels have been reported in the literature. ${ }^{1-4}$ Most of these cases are treated acutely to decompress the neural elements and optimize the clinical outcome. However, Concomitant multisystem injury often delays treatment and might affect neurological recovery. We report a case of traumatic L4-5 spondylolisthesis with neurological compromise that was treated surgically 3 months after her injury and had full recovery of her neurological status.

\section{Case report}

A 53 y.o female patient presented to the emergency of another hospital after she was involved in a motor vehicle accident and was diagnosed to have traumatic L4-5 spondylolisthesis (Figure 1). She was complaining of severe low back pain along with inability to mobilize her feet. Physical exam showed absence of motor power in both dorsiflexion and plantar flexion of both feet. There was also complete loss of sensation in all feet dermatomes. She was taken to the operating room immediately where laminectomy to decompress the neural elements was done along with repair of a massive dural tear. Several attempts to reduce the dislocation were unsuccessful and In-situ bone grafting with instrumentation between L4 and Sacrum was performed (Figure 2). Postoperatively, the right foot dorsiflexion improved to $2 / 5$ for only 8 days and then became again $0 / 5$ while the left foot did not show any sign of improvement. A brace was applied and the patient was stabilized and discharged home on physical therapy exercises for the following 3 months.

After 3 months, the patient was still complaining of persistent low back pain and inability to ambulate on her lower extremities. All treatment options and possible outcomes were thoroughly discussed with the patient including the poor prognosis regarding the neurological recovery at this point time after the accident. A second operation was done after discussing the the pros and cons and obtaining the consent of the patient. Starting with the posterior approach, Pedicle screws were inserted segmentally from L3 to S1, complete L4-5 facetectomy was done on both sides and both L4 and L5 nerve root were fully decompressed. This was followed by removal of the disc materials from both sides to help in the reduction process, which was achieved using reduction screws at L4 level with special contouring of the rods. Autologous bone graft was applied posetrolaterally. Skin incision was closed and the patient was put in a lateral decubitus position. A cage filled with bone graft was inserted at L4-5 level using the oblique lumbar interbody fusion technique (Figure 3).

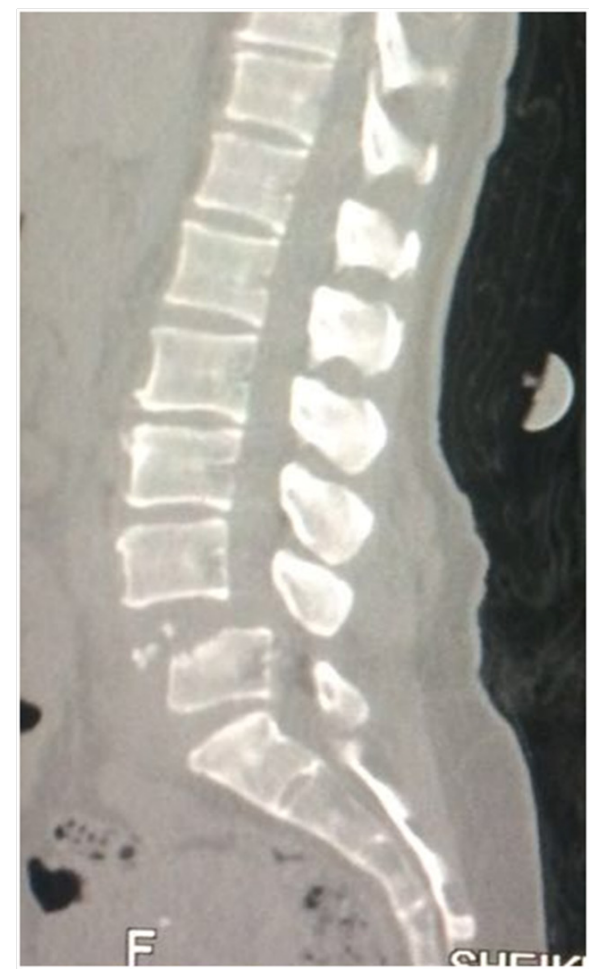

Figure I Traumatic L4-5 spondylolisthesis. 


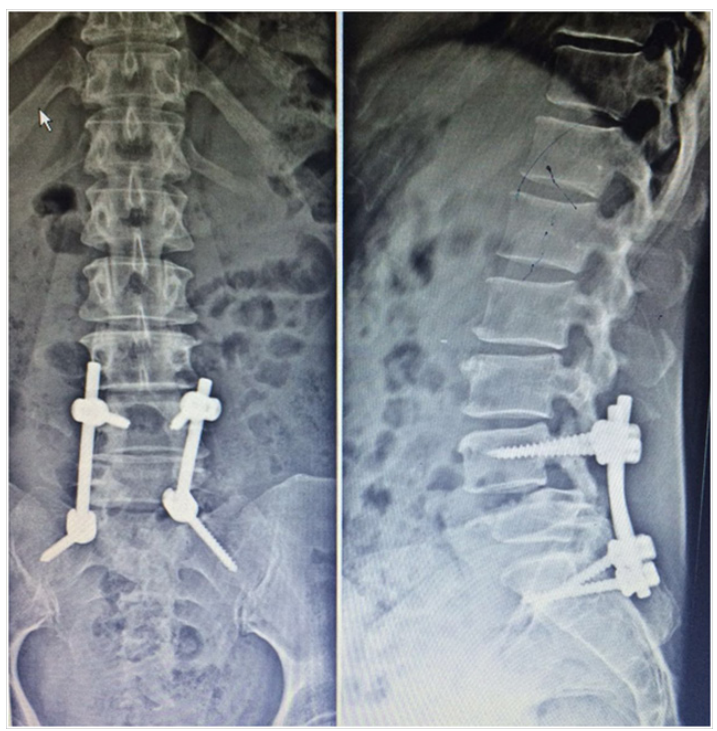

Figure 2 In-situ fusion with instrumentation between L4 and Sacrum was performed.

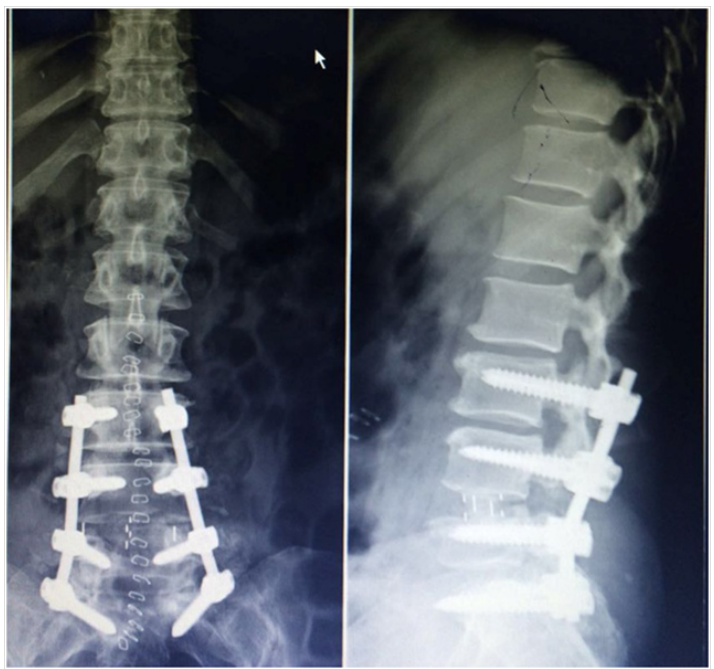

Figure 3 A cage filled with bone graft was inserted at L4-5 level using the oblique lumbar interbody fusion technique.

On the second postoperative day, the patient started to recover the motor power in her feet $(4 / 5$ on the right side and $2 / 5$ on the left side) and at 6 weeks after the surgery, the patient was able to walk independently with normal motor power in the right foot and slight weakness in the dorsiflexion of the left foot (3/5). She reported minimal back pain that was controlled by simple analgesics.

\section{Discussion}

Traumatic spondylolisthesis of the lumbar spine is an uncommon pathology reported in the literature. Most cases occur at L5-S1 level mainly due to the coronal orientation of the facet joints and the slope angle of the lumbosacral junction. Traumatic spondylolisthesis at L4-5 level occur less frequently, usually secondary to a high-energy trauma leading to unstable injury associated most of the time with neurological deficits. Different treatment options have been described in the literature but mainly decompression, reduction with posterior fusion and instrumentation along with anterior interbody cage support are the most accepted treatment modalities. Neurological recovery is unpredictable and depends mainly on the severity of injury, and timing of neural element decompression. It is well known that early decompression is associated with better recovery of neurological function.

Lim et al. ${ }^{5}$ reported on 3 cases of traumatic spondylolisthesis of the lumbar spine. Two patients underwent decompression within 24 hours and their American Spinal Injury Association impairment scale improved from $\mathrm{B}$ to $\mathrm{D}$. The third patient underwent delayed posterior decompression and instrumentation and the patient had a residual neurological deficit. They recommended early decompression with posterior stabilization for maximum recovery of neurological function. Amesiya et $\mathrm{al}^{3}{ }^{3}$ reported on a 38 yo male patient who presented with paraplegia secondary to traumatic L4-L5 spondyloptosis. The patient was stabilized and had his spine surgery 4 days after the injury. The patient started regaining motor power 48 hours after surgery and was graded as ASIA D at the last follow-up.

Not all cases with traumatic lumbar spondylolisthesis are amenable to acute surgical intervention. Since most of these cases result from a high-energy trauma, they are usually associated with mutli-system injuries that delay surgical treatment. In addition, in hospitals with limited resources, either due to lack of surgical skills or due to lack of proper instrumentation, there is a tendency either to delay treatment of these cases or to manage these complex cases with minimal surgical intervention. However, this type of treatment is usually ineffective because of the persistent instability or the incomplete decompression of the neural elements.

Several reports have been published in the literature describing delayed treatment of patients with traumatic spondylolisthesis of the lumbar spine with good clinical outcome. Bellew et al. ${ }^{6}$ described a 36 -year-old woman with traumatic lateral spondyloptosis at L-2 and complete cauda equine injury who experienced a remarkable recovery despite that her surgery was delayed for 3 weeks. Deniz et al. ${ }^{1}$ reported on a case of L4-L5 traumatic anterolisthesis that was treated surgically 4 months after the injury and the patient became symptom free after 3 months. Wangtaphan et al. ${ }^{7}$ reported on a 45 year old male patient with L5-S1 spondyloptosis who was treated conservatively for 5 months with improvement of his neurological function but with persistence of his severe low back pain. The patient underwent in situ posterior fusion and instrumentation. His lower back pain had nearly disappeared 2 weeks after surgery, and he was able to walk for more than 1 hour without assistance 2 years after surgery.

In our case, there was little hope of neurological improvement 3 months after the initial injury. However, the remarkable postoperative neurological improvement proves that operative decompression of neural elements with adequate stabilization of the spine should always be attempted irrespective of the severity or chronicity of the injury because there is always a chance of some degree of neurological recovery.

\section{Acknowledgments}

None.

\section{Conflicts of interest}

None.

\section{References}

1. Deniz FE, Zileli M, Caglı S et al. Traumatic L4-L5 spondylolisthesis: case report. Eur Spine J 2008; 17(Suppl 2):S232-S235.

2. Yadla S, Lebude B, Tender GC et al. Traumatic spondyloptosis of the thoracolumbar spine. J Neurosurg Spine. 2008;9(2):145-151. 
3. Amesiya R, Orwotho N, Nyati M et al. Traumatic spondyloptosis of the lumbar spine: a casereport. Journal of Medical Case Reports. 2014;8:453.

4. Zhou TH, Tang X, Xu YQ et al. Traumatic spondyloptosis of L4. Spine (Phila Pa 1976). 2010;35(17):E855-E859.

5. Lim CT, Hee HT, Liu G Traumatic spondylolisthesis of the lumbar spine: a report of three cases. J Orthop Surg (Hong Kong). 2009;17(3):361-365.
6. Bellew MP, Bartholomew BJ Dramatic neurological recovery with delayed correction of traumatic lumbar spondyloptosis. Case report and review of the literature. J Neurosurg Spine. 2007;6(6):606-610.

7. Wangtaphan W, Oo M, Paholpak $\mathrm{P}$ et al. Traumatic lumbosacral spondyloptosis treated five months after injury occurrence: a case report. Spine (Phila Pa 1976). 2012;37(22):E1410-E1414 